\title{
Steroid-induced psychiatric symptoms: What you need to know
}

\section{Early recognition of these symptoms is key to initiating effective interventions}

$\mathrm{M}$ s. $\mathrm{N}$, age 30 , presents to the emergency department for altered mental status, insomnia, and behavioral changes, which she has experienced for 1 week. On evaluation, she grabs a clinician's hand and details her business ideas and life story with no prompting. Ms. N's mental status examination is significant for hyperverbal speech with increased rate and volume; tangential thought process; and bright, expanded affect.

One week earlier, Ms. $\mathrm{N}$ was hospitalized for sudden-onset chest pain, weakness, and dizziness. She received 45 minutes of cardiopulmonary resuscitation prior to presentation and was found to have a ST-segment elevation myocardial infarction that required emergent left anterior descending coronary artery and right coronary artery percutaneous coronary intervention to place drug-eluting stents. Her recovery was complicated by acute cardiogenic shock, pulmonary edema, and hypoxic respiratory failure. Subsequently, she was intubated, admitted to the ICU, and received high-dose corticosteroids, including IV methylprednisolone, $40 \mathrm{mg}$ every 12 hours, which was tapered prior to discharge. Her husband reports that since Ms. $\mathrm{N}$ came home, she has been more talkative and irritable, ruminating about past events, unable to sleep ( $<1$ hour/night), and crying frequently. She has also been endorsing visual and auditory hallucinations, with increased praying and listening to religious music.

The frequent clinical use of steroids necessitates an understanding of these medications' various adverse effects.

continued

\section{Disclosures}

The authors report no financial relationships with any companies whose products are mentioned in this article, or with manufacturers of competing products.

doi: $10.12788 /$ cp.0104

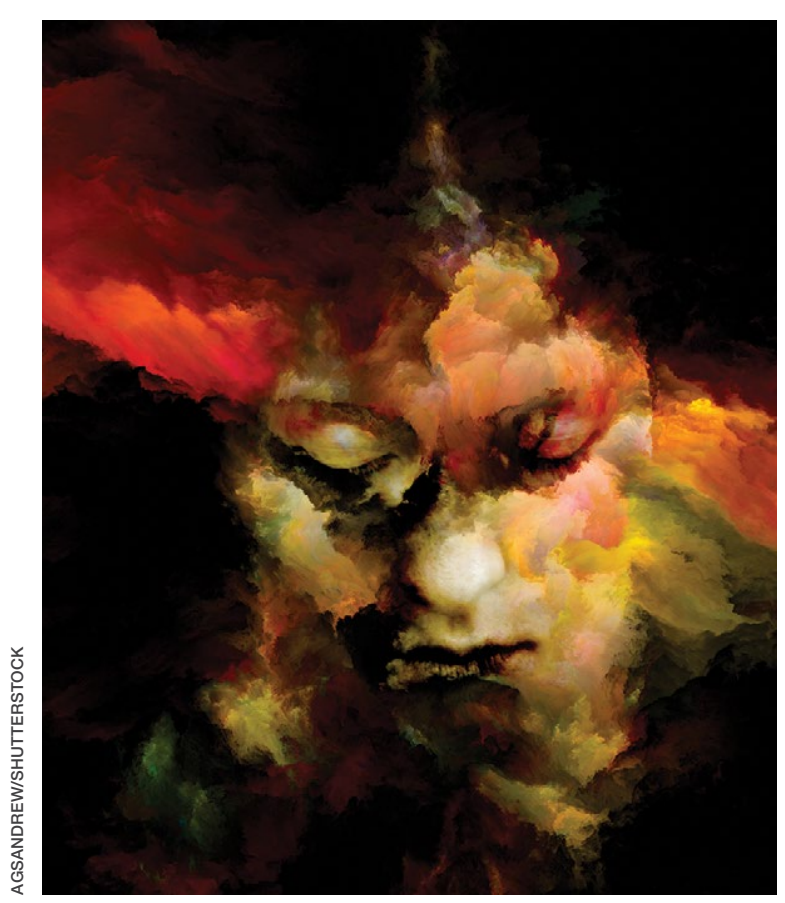

Yvonne Lu, BS

Medical Student

University of California Irvine School of Medicine Irvine, California

\section{Lydia Ann, MD}

PGY-3 Psychiatry Resident

Department of Psychiatry

University of California Irvine

University of California Medical Center

Orange, California

\section{Robert McCarron, DO}

Professor and Vice Chair

Department of Psychiatry

University of California Irvine

University of California Medical Center

Orange, California 


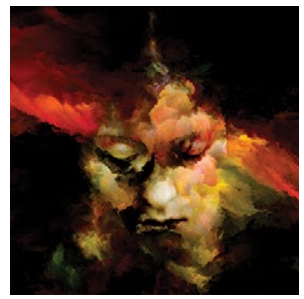

Steroids and psychopathology

\section{Clinical Point}

Mania/hypomania is the most common steroid-induced psychiatric symptom

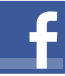

Discuss this article at www.facebook.com/ MDedgePsychiatry
The manifestations of steroid-induced psychiatric symptoms are broad and can involve affective, behavioral, and cognitive domains. While the current mechanism is unknown, this phenomenon may be related to decreased levels of corticotropin, norepinephrine, and beta-endorphin immunoreactivity, as well as effects on brain regions such as the hippocampus and amygdala. The best interventions for steroid-induced psychiatric symptoms are awareness and early diagnosis. There are no FDA-approved treatments for steroid-induced psychiatric symptoms; initial measures should include tapering or discontinuing corticosteroids.

In this article, we review the literature on the incidence, characteristics, differential diagnoses, proposed mechanism, risk factors, and proposed treatments of steroidinduced psychiatric symptoms.

\section{A wide range of presentations}

Steroid use has increased over the past 2 decades, with $10 \%$ of medical and surgical inpatients and $1 \%$ to $3 \%$ of the general population taking long-term glucocorticoids. ${ }^{1}$ Even with topical application, steroid therapy is often systemically absorbed, and thus may lead to steroid-induced psychiatric symptoms. The incidence of steroid-induced psychiatric symptoms is difficult to assess because there can be a wide range of reactions that are dose- and time-related. Three reviews of a total of 122 cases reports found that an estimated $5 \%$ of patients treated with steroids experience severe psychiatric reactions. ${ }^{1-3}$

Steroid-induced psychopathology can include mood, behavioral, and/or cognitive impairments. Mania/hypomania is the most common overall psychiatric symptom; the most common mood manifestations are anxiety and depression. ${ }^{4,5}$ Other possible steroid-induced symptoms include psychosis, dementia, panic disorder, delirium, suicidal thinking and behavior, aggressive behavior, insomnia, agitation, depersonalization, and euphoria. ${ }^{5}$ The most common cognitive impairment is verbal or declarative memory deficit; others include distractibility and deficits in attention and psychomotor speed. ${ }^{5}$ These psychiatric symptoms can have a rapid onset, possibly within hours of starting steroids. ${ }^{1}$ However, studies have reported a median time to onset of 11.5 days; $39 \%$ of cases had onset during the first week and $62 \%$ within 2 weeks. ${ }^{3,6}$ After reducing or stopping the steroid, it may take days to weeks before symptoms start to subside. ${ }^{2}$

\section{What to consider in the differential Dx}

Psychiatric symptoms that are induced by steroids can mimic metabolic, neurologic, or toxic disorders. Other factors to consider include drug withdrawal/intoxication, infections, and paraneoplastic syndromes. ${ }^{4,5}$ Although there is no reported correlation between the location of neurologic lesions and the development of specific psychiatric symptoms, manic symptoms appear most commonly with lesions in the right frontal lobe. ${ }^{4}$ Other factors to note include the presence of new-onset psychiatric illnesses such as bipolar, mood, or thought disorders, ${ }^{4}$ as well as psychosocial stressors that might be contributing to the patient's presentation. ${ }^{5}$

\section{Proposed mechanisms}

Although the exact mechanism by which steroids induce psychiatric symptoms is unknown, several mechanisms have been proposed. One hypothesis is that steroidinduced psychopathology is related to decreased levels of corticotropin, norepinephrine, and beta-endorphin immunoreactivity. ${ }^{4,5,7}$ This may explain why many patients with major depressive disorder have elevated cortisol production and/ or lack of suppression of cortisol secretion during a dexamethasone stimulation test, and why approximately one-half of patients with Cushing's disease experience depressive symptoms. ${ }^{8}$ This is also likely why antipsychotics, which typically reduce cortisol, are efficacious treatments for some steroid-induced psychiatric symptoms. ${ }^{9}$

Cognitive impairments from steroid use may be related to these agents' effects on certain brain regions. One such area is the hippocampus, an important mediator in the creation and maintenance of episodic and declarative memories. ${ }^{5,8,9}$ 
Acute glucocorticoid use is associated with decreased activity in the left hippocampus, reduced hippocampal glucose metabolism, and reduced cerebral blood flow in the posterior medial temporal lobe. ${ }^{10}$ Long-term glucocorticoid exposure is associated with smaller hippocampal volume and lower levels of temporal lobe $\mathrm{N}$-acetylaspartate, a marker of neuronal viability. ${ }^{10}$ Because working memory depends on the prefrontal cortex and declarative memory relies on the hippocampus, deficits in these functions can be attributed to the effect of prolonged glucocorticoid exposure on glucocorticoid or mineralocorticoid receptors in the hippocampus, reduction of hippocampal volume, or elevated glutamate accumulation in that area. ${ }^{11}$ In addition, high cortisol levels inhibit brain-derived neurotrophic factor, which plays a crucial role in maintaining neural architecture in key brain regions such as the hippocampus and prefrontal cortex. ${ }^{11}$ There is also a correlation between the duration of prednisone treatment and atrophy of the right amygdala, which is an important regulator of mood and anxiety. ${ }^{11}$ Both the hippocampus and amygdala have dense collections of glucocorticoid receptors. This may explain why patients who receive high-dose corticosteroids can have reversible atrophy in the hypothalamus and amygdala, leading to deficits in emotional learning and the stress response.

\section{Factors that increase risk}

Several factors can increase the risk of steroid-induced psychopathology. The most significant is the dose; higher doses are more likely to produce psychiatric symptoms. ${ }^{1,5}$ Concurrent use of drugs that increase circulating levels of corticosteroids, such as inhibitors of the cytochrome P450 (CYP) enzyme (eg, clarithromycin), also increases the likelihood of developing psychiatric symptoms. ${ }^{1,5}$ Risk is also increased in patients with liver or renal dysfunction. ${ }^{1}$ Cerebral spinal fluid/serum albumin ratio, a marker of blood-brain barrier damage, and low serum complement levels were also reported to be independent risk factors, ${ }^{12}$ with the thought that increased permeability of the blood-brain barrier may allow hydrophobic steroid molecules to more easily penetrate the CNS, leading to increased neuropsychiatric effects. Hypoalbuminemia is another reported risk factor, perhaps because lower levels of serum albumin are related to higher levels of free and active glucocorticoids, which are normally inactive when bound to albumin. ${ }^{13}$ There also appears to be an increased prevalence of steroid-induced psychopathology in women, perhaps due to greater propensity in women to seek medical care or a higher prevalence of women with medical disorders that are treated with steroids. ${ }^{5}$ A previous history of psychiatric disorders may not increase risk. ${ }^{5}$

Several methods for reducing risk have been proposed, including using a divideddosing regimens that may lower peak steroid plasma concentrations. ${ }^{13,14}$ However, the best prevention of steroid-induced psychiatric symptoms are awareness, early diagnosis, and intervention. Studies have suggested that $\mathrm{N}$-methyl-D-aspartate (NMDA) antagonists ${ }^{15}$ and other agents that decrease glutamate release (such as phenytoin and lamotrigine ${ }^{16}$ ) may help prevent corticosteroid-induced hippocampal volume loss. Lamotrigine has been shown to reduce the amount of atrophy in the amygdala in patients taking corticosteroids. ${ }^{17}$ Phenytoin has also been reported to reduce the incidence of hypomania associated with corticosteroids, perhaps due to its induction of CYP450 activity and acceleration of steroid clearance. ${ }^{16}$

\section{Treatment options}

There are no FDA-approved medications for managing steroid-induced psychiatric symptoms. ${ }^{1,16}$ Treatment is based on evidence from case reports and a few small case series (Table, ${ }^{2-5,17,18}$ page 36).

When possible, initial treatment should include discontinuing or tapering corticosteroids to $<40 \mathrm{mg} / \mathrm{d}$ of prednisone-equivalent. ${ }^{1,4,10,18}$ Most studies have reported rapid reversal of deficits in declarative memory and of hippocampal volume loss once corticosteroids were tapered and discontinued. ${ }^{1,18}$ One study reported that $>90 \%$ of patients recovered within 6 weeks, with

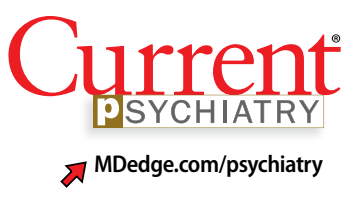

Clinical Point

Starting valproate while continuing corticosteroids may help lessen mania 


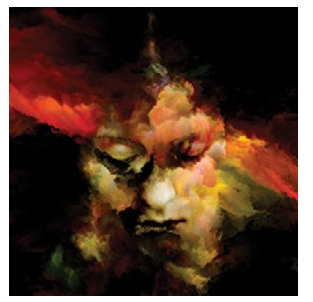

Steroids and psychopathology

\section{Clinical Point}

$\mathrm{ECT}$ is effective

for patients with

persistent and/or

unresponsive

steroid-induced

depression

\section{Table}

\section{Proposed treatments for steroid-induced psychiatric symptoms ${ }^{\mathrm{a}}$}

\begin{tabular}{|l:l}
\hline Symptom & Proposed treatments \\
\hline Mania/hypomania & $\begin{array}{l}\text { Aood stabilizers (valproate, lithium, lamotrigine) } \\
\text { a benzodiazepine }\end{array}$ \\
\hline Depression & $\begin{array}{l}\text { SSRIs (sertraline, paroxetine) } \\
\text { Antipsychotics (especially risperidone) }\end{array}$ \\
& Lithium \\
\hline Agitation & Benzodiazepines \\
& Haloperidol \\
\hline aThese proposed treatments are off-label and largely anecdotal \\
ECT: electroconvulsive therapy; SSRls: selective serotonin reuptake inhibitors \\
Source: References 2-5,17,18
\end{tabular}

patients with delirium recovering more quickly (mean: 5.4 days) than those with depression, mania, or psychosis (mean: 19.3 days). ${ }^{3}$ Another found that the vast majority $(92 \%)$ of patients treated only with a steroid taper achieved clinical recovery, and $84 \%$ recovered with administration of antipsychotics without a steroid taper. ${ }^{3}$ In this study, all patients who received electroconvulsive therapy (ECT) recovered, as did those who received a steroid taper plus lithium or antipsychotics. Steroid tapering regimens are especially important for patients who have received long-term glucocorticoid treatment. Patients need to be closely monitored for signs of new or increased depression, delirium, or confusion during the taper. If these symptoms occur, the patient should be checked for adrenocortical insufficiency, which can be resolved by re-administering or increasing the dosage of the glucocorticoid. ${ }^{10}$

Mania. The treatment of mania/hypomania includes mood stabilizers (valproate, lithium, lamotrigine) and antipsychotics (quetiapine, olanzapine, haloperidol). ${ }^{2,4,5,10,14,18}$ Valproate has been reported to be an effective prophylactic of corticosteroid-induced mania, ${ }^{2}$ perhaps because it dampens neuronal hyperexcitability by attenuating NMDA receptors, blocking voltage-dependent sodium channels, and inhibiting the synthesis of cortical GABAergic steroids. Starting valproate while continuing corticosteroids (if necessary) may help lessen mania. ${ }^{2}$ Benzodiazepines also may be useful on a short-term basis.

Depression. Steroid-induced depression may be treated with sertraline or other firstline antidepressants. ${ }^{5,14}$ Consider ECT for patients with severe depression. Support for the use of antipsychotic medications stems from studies that reported steroids' role in disrupting dopamine and 5HT2 activity. Lithium also has been used successfully to manage and prevent glucocorticoid-associated affective disorder. ${ }^{10,18}$ It can be used alone or in combination with selective serotonin reuptake inhibitors to alleviate depressive symptoms. ${ }^{10}$ Tricyclic antidepressants are generally avoided because their anticholinergic effects can exacerbate or worsen delirium. ${ }^{18}$ In general, ECT is an effective treatment for persistent and/or unresponsive steroid-induced depression, ${ }^{2,10}$ but may be difficult to use in patients with serious medical illnesses.

Agitation. Medications that have been proposed for treating steroid-induced agitation include benzodiazepines, haloperidol, and second-generation antipsychotics. ${ }^{5,17}$

Other considerations. Clinicians, patients, and families should discuss in detail the risks of steroid-induced psychiatric symptoms so an early diagnosis and appropriate intervention can be implemented. Before starting steroids, it is important to review the patient's current medication list to ensure that steroid treatment is indicated, 
and to check for potential drug-drug interactions. In addition, the medical condition that is being treated with steroids also needs to be carefully reviewed, because certain illnesses are associated with the development of psychiatric symptoms. ${ }^{5,10}$

Young children $($ age $<6)$ and older adults appear to be at greater risk for cognitive and memory disturbances from steroid use. ${ }^{10}$ In addition, patients have individual levels of susceptibility to steroid-induced psychiatric symptoms that can vary over time. The risk for adverse effects may be elevated based on response to previous courses of glucocorticoid treatment. ${ }^{10}$ While gender, age, dosage, and duration of treatment influence risk, it is not possible to predict which patients will experience psychiatric effects during a given course of glucocorticoid therapy. Therefore, all patients should be considered to have the potential of developing such effects, and should be monitored during glucocorticoid treatment and withdrawal.

\section{Goals for future research}

To help reduce the severity of and cost associated with steroid-induced psychiatric symptoms, ${ }^{5,14}$ future studies should focus on controlled trials of preventative strategies. In particular, recent advances in genetic mapping may help identify involvement of certain genes or polymorphisms. ${ }^{5}$ Because current guidelines for the prevention and treatment of steroid-induced psychiatric symptoms are not evidencebased, controlled clinical trials are needed to elucidate the optimal management of such symptoms. There is much interplay between many of the proposed mechanisms of steroid-induced psychiatric symptoms,

\section{Related Resources}

- Janes M, Kuster S, Goldson TM, et al. Steroid-induced psychosis. Proc (Bayl Univ Med Cent). 2019;32(4):614-615.

- Mayo Clinic. Prednisone and other corticosteroids. https:// www.mayoclinic.org/steroids/art-20045692

Drug Brand Names

Haloperidol · Haldol

Lamotrigine - Lamictal

Paroxetine $\cdot$ Paxil

Lithium • Eskalith, Lithobid

Phenytoin - Dilantin

Methylprednisolone

injection - Solu-Medrol

Quetiapine · Seroquel

Risperidone $\cdot$ Risperda

Olanzapine $\cdot$ Zyprexa

Sertraline $\cdot$ Zoloft

Valproate - Depakote

and future studies can help uncover a deeper understanding of the intricacies of this phenomenon.

\section{CASE CONTINUED}

Mrs. $\mathrm{N}$ is admitted for altered mental status. Medical workup includes MRI of the brain, MRI of the neck, cardiac echocardiogram, and EEG. There is no evidence of acute structural pathology. She is started on olanzapine, $10 \mathrm{mg} / \mathrm{d}$ at bedtime for manic and psychotic symptoms, and is discharged after 5 days. After 1 month, the outpatient psychiatrist gradually decreases and discontinues olanzapine as Mrs. $\mathrm{N}$ steadily returns to baseline. One year after discharge, Mrs. $\mathrm{N}$ continues to report resolution of her manic and psychotic symptoms.

\section{References}

1. Dubovsky AN, Arvikar S, Stern TA, et al. The neuropsychiatric complications of glucocorticoid use: steroid psychosis revisited. Psychosomatics. 2012;53(2): 103-115.

2. Roxanas MG, Hunt GE. Rapid reversal of corticosteroidinduced mania with sodium valproate: a case series of 20 patients. Psychosomatics. 2012;53(6):575-581.

3. Lewis DA, Smith RE. Steroid-induced psychiatric syndromes. A report of 14 cases and a review of the literature. J Affect Disord. 1983;5(4):319-332.

4. Warren KN, Katakam J, Espiridion ED. Acute-onset mania in a patient with non-small cell lung cancer. Cureus. 2019;11(8):e5436.

continued

\section{Bottom Line}

Steroids can induce a wide range of psychiatric symptoms, including mania/ hypomania, anxiety, and depression. Initial treatment typically includes tapering or discontinuing the steroid when possible. Other proposed treatments include certain antipsychotics, antidepressants, and other psychotropics, but the supporting evidence is largely anecdotal or based on case studies. Additional research is needed to elucidate the mechanism and treatment recommendations.

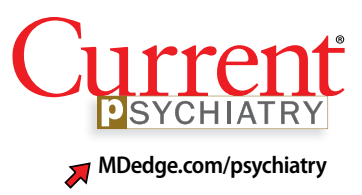

Clinical Point

Benzodiazepines, haloperidol, and second-generation antipsychotics have been used to treat steroid-induced agitation 


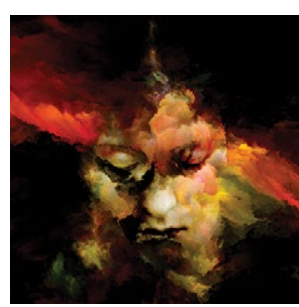

Steroids and psychopathology

\section{Clinical Point}

While certain patients are at higher risk, all patients should be considered to have the potential to develop steroid-induced psychiatric symptoms
5. Kenna HA, Poon AW, de los Angeles CP, et al. Psychiatric complications of treatment with corticosteroids: review with case report. Psychiatry Clin Neurosci. 2011;65(6):549-560

6. Ling MH, Perry PJ, Tsuang MT. Side effects of corticosteroid therapy. Psychiatric aspects. Arch Gen. Psychiatry. 1981;38(4): 471-477.

7. Ularntinon S, Tzuang D, Dahl G, et al. Concurrent treatment of steroid-related mood and psychotic symptoms with risperidone. Pediatrics. 2010;125(5):e1241-e1245.

8. Pokladinkova J, Meyboom RH, Vlcek J, et al. Intranasally administered corticosteroids and neuropsychiatric disturbances: a review of the international pharmacovigilance programme of the World Health Organization. Ann Allergy Asthma Immunol. 2008;101(1):67-73.

9. Walker EF, Trotman HD, Pearce BD, et al. Cortisol levels and risk for psychosis: initial findings from the North American prodrome longitudinal study. Biol Psychiatry. 2013;74(6): 410-417.

10. Wolkowitz OM, Reus UI. Treatment of depression with antiglucocorticoid drugs. Psychosom Med. 1999;61(5): 698-711.

11. Judd LL, Schettler PJ, Brown ES, et al. Adverse consequences of glucocorticoid medication: psychological, cognitive, and behavioral effects. Am J Psychiatry. 2014;171(10):1045-1051.

12. Appenzeller S, Cendes F, Costallat LT. Acute psychosis in systemic lupus erythematosus. Rheumatol Int. 2008, 28(3):237-243

13. Glynne-Jones R, Vernon CC, Bell G. Is steroid psychosis preventable by divided doses? Lancet. 1986;2(8520):1404

14. Ismail MF, Lavelle C, Cassidy EM. Steroid-induced mental disorders in cancer patients: a systematic review. Future Oncol. 2017;13(29):2719-2731.

15. Magariños AM, McEwen BS. Stress-induced atrophy of apical dendrites of hippocampal CA3c neurons: involvement of glucocorticoid secretion and excitatory amino acid receptors. Neuroscience. 1995;69(1):89-98.

16. Brown BS, Stuard G, Liggin JDM, et al. Effect of phenytoin on mood and declarative memory during prescription corticosteroid therapy. Biol Psychiatry. 2005;57(5): 543-548.

17. Desai S, Khanani S, Shad MU, et al. Attenutation of amygdala atrophy with lamotrigine in patients receiving corticosteroid therapy. J Clin Psychopharmacol. 2009;29(3):284-287.

18. Gable M, Depry D. Sustained corticosteroid-induced mania and psychosis despite cessation: a case study and brief literature review. Int J Psychiatry Med. 2015;50(4):398-404. 